Case Report

\title{
A dangerous surgical masquerade - medial arcuate ligament syndrome as acute coronary syndrome: a case report
}

\author{
Preethi Subramanian, Rajan Vaithianathan*
}

Department of Surgery, Mahatma Gandhi Medical College, Pondicherry, India

Received: 17 February 2020

Accepted: 08 April 2020

\section{*Correspondence:}

Dr. Rajan Vaithianathan,

E-mail: 57sylvan@gmail.com

Copyright: ( $)$ the author(s), publisher and licensee Medip Academy. This is an open-access article distributed under the terms of the Creative Commons Attribution Non-Commercial License, which permits unrestricted non-commercial use, distribution, and reproduction in any medium, provided the original work is properly cited.

\begin{abstract}
Median arcuate ligament syndrome is an uncommon cause for abdominal pain and weight loss, caused by median arcuate ligament compressing the celiac plexus or artery. Median arcuate ligament is the continuation of the posterior diaphragm which passes superior to celiac artery and surrounds the aorta. In this case report, A 67 year old male presented with complaints of sudden onset chest pain and loss of weight for the past 6 months. CECT thorax and abdomen it showed features of focal stenosis of coeliac axis and post stenotic dilation of the coeliac trunk suggesting median arcuate ligament syndrome. Laparoscopic median arcuate ligament release was done to relieve the patient from symptoms. Diagnosis of median arcuate ligament syndrome should be considered in a patient presenting with chest pain and weight loss with normal cardiac status and unexplained etiology.
\end{abstract}

Keywords: Celiac artery stenosis, Laparoscopy, Median arcuate ligament

\section{INTRODUCTION}

The median arcuate ligament is a fibrous arch that arises from crura of diaphragm on either side of aorta. It passes superior to the origin of the celiac artery. Median arcuate ligament syndrome also known by other names such as celiac axis compression syndrome and Dunbar's syndrome is a rare condition caused by the external compression of the celiac plexus or artery by the median arcuate ligament.

It is characterized by a triad of epigastric pain, loss of weight and post prandial pain with emesis. ${ }^{1}$ The same condition can also present with non-specific symptoms such as retrosternal chest mimicking cardiac cause. CECT is the investigation of choice which will show focal narrowing and hooked appearance in the proximal celiac axis. We report a patient with median arcuate ligament syndrome presenting with sudden onset of chest pain which was successfully treated with laparoscopic median arcuate ligament release.

\section{CASE REPORT}

A 67 year old male presented with complaints of sudden onset chest pain and a significant loss of weight over the past 6 months.

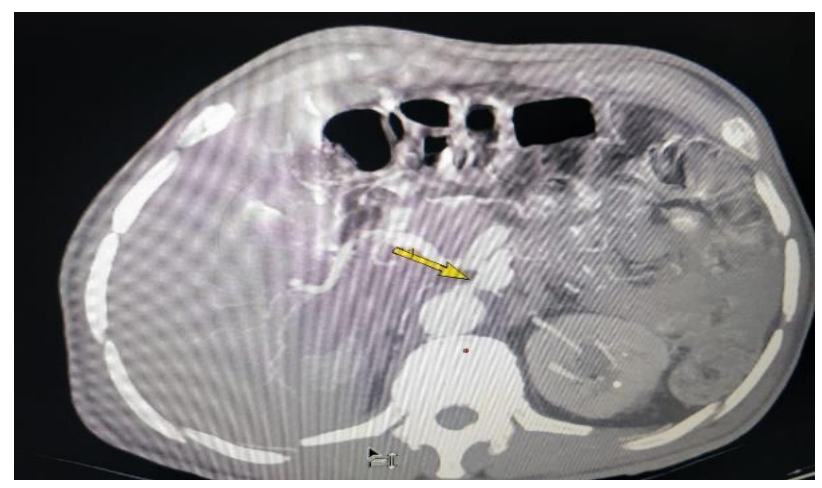

Figure 1: CT celiac angiogram showing post stenotic dilation of celiac artery. 
He was admitted in cardiology under the suspicion of acute coronary syndrome. Complete cardiac workup revealed no significant abnormality following which Upper GI endoscopy also showed no significant abnormality. On proceeding with CECT thorax and abdomen to rule out aotic dissection and aneurysm, it showed features of focal stenosis of coeliac axis and post stenotic dilation of the coeliac trunk suggesting median arcuate ligament syndrome (MALS). The patient was then transferred to surgery department. Laparoscopic median arcuate ligament was done. Post-operative period was uneventful and the patient was relieved of his symptoms.

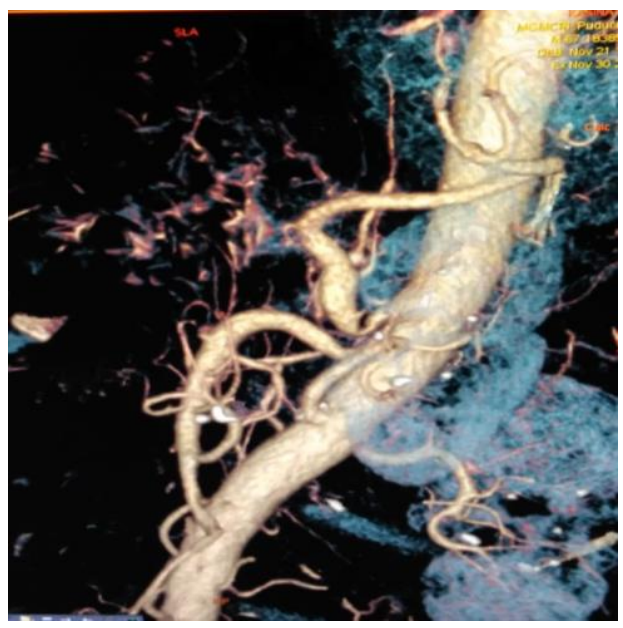

Figure 2: 3D reconstruction of celiac axis with stenosis.

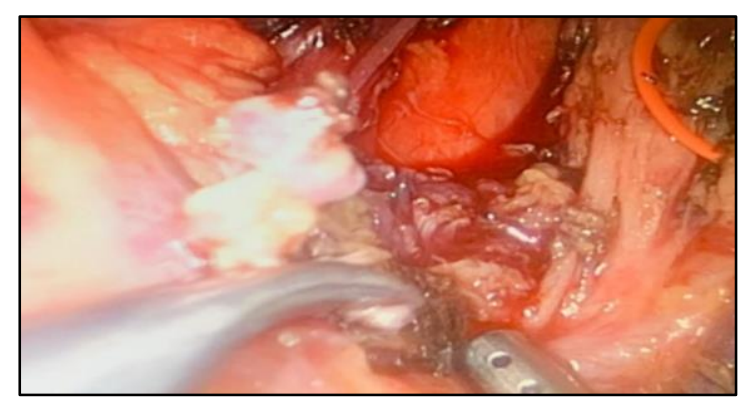

Figure 3: Operative picture showing dissection of celiac trunk.

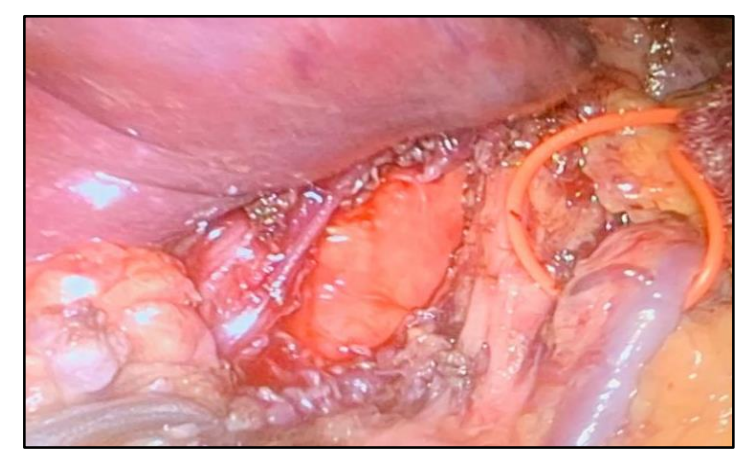

Figure 4: Division of median arcuate ligament.

\section{DISCUSSION}

Median arcuate ligament syndrome is characterized by abdominal pain caused due to compression of the celiac artery and occasionally the celiac ganglia by the median arcuate ligament. Harjola in 1963 was the first to describe MALS. ${ }^{2}$ Later in 1965, Dunbar in his case series reported successful treatment of MALS. ${ }^{3}$ Thus the other name of Dunbar syndrome. The anatomical abnormality in median arcutate ligament especially low lying ligament which causes compression leading to compromise in blood flow which causes the symptoms. Some patients might be asymptomatic for a prolonged period due to collateral circulation. MALS is frequently seen in young females with thin body and a classical triad of epigastric pain, loss of weight and post prandial pain with emesis. CECT and angiography are the choice of investigation for diagnosis of MALS. Treatment of this condition is surgical or laparoscopic division of the median arcuate ligament. In 1984 a study by Reilly is still considered the largest series of open surgical patients for MALS. ${ }^{4}$ In 2010 a study by Tulloch concluded that both laparoscopic and open MAL division along with celiac ganglionectomy can be safely performed. ${ }^{5}$ Relief from symptoms is usually immediate and can take upto a month to resolve following a successful procedure. Still if patient is symptomatic they are treated by angioplasty with stenting. ${ }^{6}$

\section{CONCLUSION}

MALS is a rare condition and diagnosis is difficult in majority of cases. MALS patient may present with symptoms outside of the triad which could make diagnosis even difficult as our patient presented with sudden onset of chest pain mimicking acute coronary syndrome. Confirmation of this diagnosis can be performed by CT angiography. Once MALS is diagnosed patient should undergo surgical decompression either open or laparoscopically.

\section{Funding: No funding sources Conflict of interest: None declared Ethical approval: Not required}

\section{REFERENCES}

1. Cienfuegos JA, Rotellar F, Valenti V, Arredondo J, Pedano N, Bueno A, et al. The celiac axis compression syndrome (CACS): critical review in the laparoscopic era. Rev Esp Enfern Dig. 2010;102:193-201.

2. Harjola PT. A rare obstruction of the coeliac artery. Report of a case. Annales Chirurgiae et Gynaecologiae Fenniae. 1963;52:547-50.

3. Dunbar JD, Molnar W, Beman FF, Marable SA. Compression of the celiac trunk and abdominal angina. Am J Roentgenol Radium Therap Nuclear Med. 1965;95(3):731-44.

4. Reilly LM, Ammar AD, Stoney RJ, Ehrenfeld WK. Late results following operative repair for celiac 
artery compression syndrome. J Vasc Surg. 1985;2(1):79-91.

5. Tulloch AW, Jimerez JC, Lawrence PF, Dutson EP, Moore WS, Rigberg DA, et al. Laparscopic versus open celiac ganglionectomy in patients with median arcuate ligament syndrome. J Vasc Surg. 2010;52(5):1283-9.

6. Duffy AJ, Panait L, Eisenberg D, Bell RL, Roberts KE, Sumpio B. Management of median arcuate ligament syndrome: a new paradigm. Ann Vasc Surg. 2009;23(6):778-84.

Cite this article as: Subramanian $\mathrm{P}$, Vaithianathan R. A dangerous surgical masquerade - medial arcuate ligament syndrome as acute coronary syndrome: a case report. Int Surg J 2020;7:2016-8. 